\title{
Postmortem angiography using femoral cannulation and postmortem microbiology
}

\author{
Cristian Palmiere • Coraline Egger • Silke Grabherr • \\ Katia Jaton-Ogay • Gilbert Greub
}

Received: 4 May 2014 / Accepted: 29 October 2014 / Published online: 8 November 2014

(C) Springer-Verlag Berlin Heidelberg 2014

\begin{abstract}
Despite the undeniable advantages of postmortem angiography, numerous questions have arisen concerning the influence that the injected contrast media may exercise on biological fluids and tissues collected for toxicological and biochemical investigations. Moreover, cardiac blood for microbiological investigations cannot be obtained post-angiography. In this study, we examined whether the peripheral blood collected prior to postmortem angiography, using percutaneous access to femoral vessels after skin surface disinfection, could be suitable for microbiological investigations when postmortem angiography with femoral vessel cannulation is also performed. A total of 66 cases were included in the study and were divided into two subgroups (angiography and bacteriology group, 33 cases and control group, 33 cases). Autopsies, histology, toxicology, bacteriology, and biochemical investigations (procalcitonin, C-reactive protein, interleukin-6, and soluble triggering receptors expressed on myeloid cells type 1) were performed in all cases. No statistically significant differences between the two groups were noted, and identified category distribution (death unrelated to infection, true infection, false positive, and undetermined) was rather similar in both studied populations. These preliminary results suggest that postmortem angiography using a femoral approach does not constitute an impediment to the collection
\end{abstract}

C. Palmiere $(\bowtie) \cdot$ C. Egger $\cdot$ S. Grabherr

University Centre of Legal Medicine, University Hospital Center and

University of Lausanne, Rue du Bugnon 21, 1011 Lausanne,

Switzerland

e-mail: cristian.palmiere@chuv.ch

K. Jaton-Ogay $\cdot$ G. Greub

Institute of Microbiology, University Hospital Center and University

of Lausanne, Lausanne, Switzerland

G. Greub

Service of Infectious Diseases, University Hospital Center,

Lausanne, Switzerland of peripheral blood for microbiology and vice versa. Moreover, the use of femoral blood for microbiology does not lead to an increased risk of doubtful results.

Keywords Postmortem bacteriology $\cdot$ Postmortem biochemistry $\cdot$ Postmortem angiography $\cdot$ Infection $\cdot$ Blood cultures

\section{Introduction}

Postmortem angiography has become a key imaging in the investigation and identification of bleeding sources in cases of acute hemorrhages with fatal outcomes, especially when affected vessels are of small caliber or located in anatomical areas of the body that are difficult to access. Despite the undeniable advantages of this technique, numerous questions have arisen concerning the influence that the injected contrast media may exercise on biological fluids and tissues collected during autopsy for toxicological and biochemical investigations [1].

It has been demonstrated that the injection of a specific contrast agent mixture (Angiofil ${ }^{\mathrm{TM}}$ ) fails to affect vitreous humor composition and measurements of specific compounds (vitreous glucose, sodium, chloride, urea nitrogen, creatinine, and beta-hydroxybutyrate) [2]. A succeeding study has shown that targeted postmortem computed tomography angiography using Urografin does not have any diagnostic effect on toxicological and biochemical investigations performed on femoral blood and vitreous humor [3].

Postmortem microbiological analyses in cardiac blood and tissue samples collected during autopsy are routinely performed in cases of sudden, unexpected deaths involving infants, young children, and young adults as well as in those suggesting bacterial infection and sepsis related-deaths [4-11]. Cardiac blood for microbiology is normally collected 
during autopsy from the right atrium post pericardium incision: the external side of the heart is sterilized by searing with a heated scalpel blade and blood is aspirated using a sterile syringe. Analogously, tissue samples are obtained by searing a small surface area of an organ to dryness with a red hot metal instrument. The area is then pierced with a sterile swab or pieces of tissue removed with sterile instruments $[12,13]$.

Cardiac blood for microbiological investigations cannot be obtained post angiography. Indeed, contrast agents used in postmortem imaging are usually not sterile and, even when sterile, render cardiac blood unavailable once it has been infused and reaches the heart. On the other hand, cardiac blood for microbiology could theoretically be obtained prior to angiography using percutaneous procedures under CTguidance, though these sampling approaches are not routinely used due to their invasiveness and subsequent risk of coronary artery damage. Indeed, the latter might create artifacts during the arterial, venous, and dynamic phases of angiography. In addition, pericardial fluid would be unavoidably mixed with cardiac blood following cardiac puncture and no longer useful.

Given the above, effort has been devoted to developing sampling procedures that allow femoral vessel cannulation to be performed at the same time that femoral blood for postmortem bacteriology, biochemistry, and toxicology is collected. Indeed, these protocols would provide the use of aseptic techniques for femoral blood sampling as well as collection of sufficient amounts of blood for microbiology, biochemistry, and toxicology to be obtained. Furthermore, femoral vessel cannulation and contrast agent injection for angiography would be able to be carried out as usual.

The aim of this study was to assess whether the peripheral blood collected prior to postmortem angiography, using percutaneous access to femoral vessels after skin surface disinfection, could be suitable for microbiological investigations when postmortem angiography with femoral vessel cannulation is also performed.

\section{Material and methods}

\section{Study design}

The present study was a prospective, single-center study, performed at the University Centre of Legal Medicine in Lausanne, Switzerland, during 2008-2013. All cases collected for this study underwent medicolegal autopsies as requested by the inquiring authorities. Postmortem microbiological analyses and biochemical investigations were performed as part of the medicolegal investigations. All cases included in this study originated from forensic practice with deaths occurring outside the hospital. Laboratory results pertaining to the period immediately preceding the death were therefore unavailable.

Study population and postmortem investigations

A total of 66 cases were included in the study. The first group (angiography and bacteriology group, ABG) consisted of 33 cases ( 20 males, 13 females) with a mean age of 51.6 years (range 19-76 years).

Postmortem angiographies were performed according to the standardized protocol of multiphase postmortem computed tomography angiography [14].

The control group (bacteriology group, BG) consisted of 33 age-, race-, gender-matched autopsy cases (20 males, 13 females; mean age 52 years, range $20-78$ years).

Intervals between deaths and autopsies ranged from 6 to $62 \mathrm{~h}$.

In all cases included in the study, an unenhanced computed tomography (CT) scan was carried out before any manipulation of the corpse. Postmortem bacteriology and biochemistry were performed as part of routine forensic investigations in suspected infection-related deaths.

Postmortem angiographies were systematically performed after CT scan and prior to autopsy.

Complete, conventional, medicolegal autopsies, histology, toxicology, bacteriology, and biochemical investigations were performed in all cases. Autopsies were jointly performed by two forensic pathologists (at least one board-certified) as in accordance with both local standards and international guidelines for medicolegal cases. Biochemical investigations were performed in postmortem serum obtained from femoral blood in all cases included in the study. These analyses consisted of measuring procalcitonin (PCT), C-reactive protein (CRP), interleukin-6 (IL-6), and soluble triggering receptors expressed on myeloid cells type 1 (sTREM-1).

\section{Sample collection}

In the first group (ABG), femoral blood for microbiology, biochemistry, and toxicology was collected from the femoral vessels after skin disinfection in the inguinal area. A chemical complex of polyvinylpyrrolidone (povidone) and elemental iodine (povidone-iodine, brand name Betadine ${ }^{\circledR}$ ) was used for disinfection. No skin incision was carried out in any of these cases, and blood was aspirated through the skin using sterile needles and syringes.

Once collected, a portion of the aspirated blood was injected into blood culture bottles, transported promptly to the laboratory for incubation at $37^{\circ} \mathrm{C}$ and cultured for aerobic and anaerobic microorganisms.

The remaining part was allotted as follows: a portion was stored in tubes containing gel serum separator and centrifuged immediately post collection at $3000 \mathrm{~g}$ for $15 \mathrm{~min}$. After 
centrifugation, the separated supernatant (postmortem serum) was collected and stored in preservative-free tubes. All these samples were transferred to the laboratories immediately post collection. When analyses were delayed, samples were stored at $-20{ }^{\circ} \mathrm{C}$.

A further portion was stored in tubes containing sodium fluoride and subsequently forwarded to the toxicology laboratory.

No cases were excluded due to insufficient sample volume.

Unilateral cannulation of the femoral vessels was achieved on the contralateral inguinal region.

In the second group (BG), femoral blood samples were collected from the femoral vein(s) immediately prior to autopsy. Blood was drawn after clamping the vein(s) at the proximal end and lifting the lower limb(s) for several minutes and subsequently collected by aspiration using a sterile needle and syringe. Blood was allotted as follows: a portion was stored in tubes containing gel serum separator and centrifuged immediately post collection at $3000 \mathrm{~g}$ for $15 \mathrm{~min}$. After centrifugation, the separated supernatant (postmortem serum, between 1 and $5 \mathrm{ml}$ ) was collected and stored in preservative-free tubes. All these samples were transferred to the laboratories immediately post collection. When analyses were delayed, samples were stored at $-20^{\circ} \mathrm{C}$.

A further portion was stored in tubes containing sodium fluoride and forwarded subsequently to the toxicology laboratory.

No specimens were excluded due to insufficient sample volume.

Cardiac blood for postmortem microbiological investigation was obtained from the heart during autopsy. The external side of the right atrium was sterilized by searing with a heated scalpel blade and cardiac blood was aspirated using a sterile needle and syringe prior to any other manipulation of either thoracic or abdominal organs. Once collected, blood was injected into blood culture bottles, transported promptly to the laboratory for incubation at $37^{\circ} \mathrm{C}$, and cultured for aerobic and anaerobic microorganisms. Tissue samples for microbiology were obtained from the spleen, the liver, and the lungs in the usual way by searing a small surface area of the organs to dryness with a red hot metal instrument and removing tissue blocks with sterile instruments. Once collected, these samples were immediately transported to the laboratory and cultured for aerobic and anaerobic microorganisms.

\section{Laboratory assays}

Cultures were performed by current standard accepted procedures. PCT, CRP, IL-6, and sTREM-1 levels were determined according to the techniques previously described $[15,16]$. Results were expressed in $\mu \mathrm{g} / \mathrm{l}, \mathrm{mg} / \mathrm{l}, \mathrm{pg} / \mathrm{ml}$, and $\mathrm{pg} / \mathrm{ml}$, respectively. Increased postmortem serum PCT, CRP, and sTREM-1 levels suggesting the presence of generalized inflammation and bacterial infections were chosen based on former medicolegal investigation results $(2 \mu \mathrm{g} / \mathrm{l}, 10 \mathrm{mg} / \mathrm{l}$, and $90 \mathrm{pg} / \mathrm{ml}$, respectively) [15]. Based on the results reported by Llewelyn et al. [17], IL-6 cutoff value was set at 200 pg/ml.

\section{Statistical analysis}

Unpaired $t$ test was used to calculate the $p$ value and determine whether significant differences existed between the two groups. Statistical significance was set at $p<0.05$.

\section{Ethics}

All relevant ethical issues were identified and discussed with the local ethical committee. All cases collected for this study underwent medicolegal autopsies as requested by the public prosecutor. Femoral blood, postmortem serum from femoral blood, and cardiac blood are systematically collected in our facility prior to or during autopsy. Moreover, biochemical investigations are routinely performed. All blood and postmortem serum samples were anonymized prior to analysis. No further ethical approval was necessary to perform microbiological and biochemical investigations in the cases included in this study.

\section{Results}

Table 1 summarizes the main findings obtained in both studied groups. Regardless of information provided by the police when the corpses were admitted to the medicolegal centre or postmortem investigation findings, the results were initially classified based on the following parameters:

- Increases in PCT, CRP, IL-6, and sTREM-1 concentrations consistent with the presence of inflammation/ generalized or localized bacterial infection,

- Number of isolated bacteria (one or more than one),

- Type of isolated bacteria (i.e., part of the normal human flora, commensal of the skin, commensal of the gastrointestinal tract, opportunistic pathogen, pathogenic in patients with compromised immune system, pathogen in nosocomial infections, potential pathogen, etc.),

- Accordance or discordance between postmortem biochemistry and microbiological analysis results.

After combining postmortem imaging data, autopsy, and histology findings as well as laboratory test results, the following situations were identified: 
Table 1 Summarizes subcategories distribution in both studies groups

\begin{tabular}{|c|c|c|c|c|c|c|c|c|}
\hline & Undetermined & $\begin{array}{l}\text { Infection } \\
\text { (one isolate) }\end{array}$ & $\begin{array}{l}\text { Infection } \\
\text { (multiple } \\
\text { isolates) }\end{array}$ & Sterile & $\begin{array}{l}\text { Contamination } \\
\text { (one isolate) }\end{array}$ & $\begin{array}{l}\text { Contamination } \\
\text { (multiple isolates) }\end{array}$ & $\begin{array}{l}\text { Translocation } \\
\text { (one isolate) }\end{array}$ & $\begin{array}{l}\text { Translocation } \\
\text { (multiple isolates) }\end{array}$ \\
\hline $\begin{array}{c}\text { ABG }(33 \\
\text { cases })\end{array}$ & 2 & 7 & 2 & 7 & 4 & 3 & 4 & 4 \\
\hline $\begin{array}{l}\text { BG }(33 \\
\text { cases })\end{array}$ & 2 & 5 & 6 & 6 & 3 & 3 & 3 & 5 \\
\hline
\end{tabular}

$A B G$ angiography and bacteriology group, $B G$ bacteriology group

- Death unrelated to infection: these cases were characterized by normal levels of biochemical markers, negative bacterial culture as well as negative autopsy and histology findings,

- True infection: these cases were characterized by increased biochemical marker levels, positive cultures for one microorganism (or more than one microorganism but at least one potentially responsible for infection) as well as autopsy and histology findings consistent with the presence of bacterial infections,

- False positive: these cases were characterized by normal levels of biochemical markers, negative postmortem investigation findings, and positive bacterial culture. Based on the isolated microorganism(s), three subcategories were identified:

(a) Contamination during sampling procedures (the isolated microorganism(s) belonged to the resident bacterial skin flora; coagulase-negative staphylococci were cultured in the most cases),

(b) Postmortem bacterial translocation (the isolated bacteria were commensals of the gastrointestinal tract in most cases),

(c) Mixed situations (mixed microorganisms suggesting combinations of postmortem translocation and contamination during sampling procedures),

- Undetermined (contradiction/incoherence among autopsy, histology, postmortem biochemistry, and microbiological analysis results).

No statistically significant differences between the two groups were noted and identified category distribution was rather similar in both studied populations. When specifically examining the proportion of cases classified as contaminated during sampling procedures in each of the two groups, we found that in 4 out of $33 \mathrm{ABG}$ cases, one single microorganism could be identified (Staphylococcus epidermidis or another coagulase-negative Staphylococcus such as Staphylococcus hominis, Staphylococcus capitis, Staphylococcus warneri, Staphylococcus cohnii, Staphylococcus pettenkoferi, Staphylococcus haemolyticus, Staphylococcus simulans, or Staphylococcus saprophyticus). In three other cases classified as contaminated during sampling procedures observed in the $\mathrm{ABG}$, two or more microorganisms were cultured. These were predominately coagulase-negative staphylococci and very occasionally belonged to other families of bacteria resident on the skin (Micrococcus, Corynebacteria, Propionibacteria).

Very similar findings in both categories (single and multiple contamination during sampling procedures) were obtained in the BG.

Bacterial translocation ( 8 out of 33 cases in both the ABG and $\mathrm{BG}$ groups) was characterized by the presence of a single (4 out of 33 cases and 3 out of 33 cases, respectively) or multiple ( 4 out of 33 cases and 5 out of 33 cases, respectively) microorganism(s) originating from the gastrointestinal tract. Gram-negative members of the Enterobacterieaceae family (Escherichia, Enterobacter, and Klebsiella) and/or Grampositive commensal(s) of the human gastrointestinal tract (mainly members of the Enterococcus groups) were the most commonly cultured bacteria.

These preliminary results suggest that percutaneous access to femoral vessels after skin disinfection of the inguinal area do not lead to an increased detection rate of contaminated blood samples. The percutaneous approach could therefore be considered a safe, alternative technique of blood sampling should both postmortem bacteriological tests and postmortem angiography using femoral access be indicated.

\section{Discussion}

Postmortem diagnosis of antemortem bacterial infection by means of blood and tissue cultures from cadaver-obtained samples is a major concern in both clinical and forensic pathology. Still, interesting data pertaining to positive blood/ tissue culture rates and isolated microorganisms can be obtained from orthopedic research and investigations carried out in other surgical fields that use tissues excised from cadaver donors for transplantation.

In a study performed by Martinez et al. [18], microbiological cultures were performed on blood and bone marrow in a series of bone-cadaver donors (with no history of sepsis or neoplastic disease at the time of death) and heart-beating donors (selected for kidney and bone excision). In the first 
group of donors, blood samples for cultures were obtained immediately prior to bone excision by cannulation of the saphenous vein or, occasionally, the femoral vein, through a cutaneous incision after skin surface decontamination with povidone iodine solution. Bone allografts were excised in a sterile operating room within $2-30 \mathrm{~h}$ of the estimated time of death. These authors observed that the incidence of positive blood cultures was significantly lower among the heartbeating donors compared to cadaver bone donors. Coagulase-negative species of staphylococci were the most common organisms isolated from blood and bone in both donor categories. Species of Propionibacterium were the second most common organisms isolated from the bones of individuals in both donor categories, followed by various species of Clostridium, including Clostridium perfringens, Clostridium sordellii, Clostridium bifermentans, Clostridium sporogenes, Clostridium cadaveris, Clostridium septicum, and Clostridium butyricum.

Comparable findings have been obtained in more recent years by other researchers. Ibrahim et al. [19] reviewed the culture results of a series of blood samples (from either central or peripheral vessels after skin surface disinfection with iodine solution) and bone allografts originating from 119 cadaveric donors. Most positive samples cultured coagulase-negative staphylococci in both blood and bone allografts, followed by species of Streptococcus (in blood) and Bacillus (in bone allografts).

Analogously, Varettas [20] analyzed the positive culture rate of allograft musculoskeletal tissue samples from 389 cadaveric donors. These authors observed that the most frequently isolated bacteria were Gram-positive cocci, especially coagulase-negative staphylococci and Streptococcus species, followed by Gram-positive bacilli, predominantly Bacillus sp., Clostridium sp., Propionibacterium sp., and Corynebacterium sp.

It must be noticed that overall positive culture rates (i.e., contamination rate) show quite large variations within studies focusing on allograft tissues, ranging from 25.1 (Varettas) to $66.3 \%$ (Martinez et al.) $[18,20]$. Interestingly, in a study performed by Bettin et al. [21], the overall contamination rate was $49 \%$, with a greater number of pathogenic microorganisms $(60 \%)$ in grafts retrieved in a morgue environment than under surgical conditions.

Obeng et al. [22] reviewed and analyzed the number of cadaveric allograft discarded as the result of positive microbiology results in a series of 1112 donors. Discards in 54 cases were due to positive skin cultures. The most frequently isolated microorganisms were S. epidermidis and Escherichia coli among Gram-positive and the Gram-negative bacteria, respectively.

Babb et al. [23] investigated the microorganisms present on the cutaneous surfaces of cadavers before and after necropsy. Specimens were obtained from the thorax, abdomen, and thighs. The results of this study revealed that the bacteria colonizing the epidermis prior to autopsy were quite similar to those found in normal, healthy skin. Isolated bacteria were largely coagulase-negative staphylococci and corynebacteria. Gram-negative bacilli, Staphylococcus aureus, and streptococci were all occasionally isolated, particularly on damaged or moist skin. The authors observed that the number of Gramnegative bacilli rapidly increased during autopsy and was not appreciably reduced even after cleaning the corpses. Isolated Gram-negative bacilli included E. coli, Klebsiella spp., Acinetobacter calcoaceticus, Proteus spp., Enterobacter spp., Citrobacter spp., Pseudomonas aeruginosa, Serratia marcescens, and Flavobacterium spp.

Søgaard et al. [24] studied the influence that organ manipulation during autopsy may have on lung and spleen tissue culture results. They observed a considerable difference between the results obtained from tissues sampled with the organs in situ compared to those obtained from tissues sampled after the organ block had been removed, leading to the conclusion that specimens for microbiological investigations should be collected preferentially with the organs in situ. The results of Søgaard et al. [24] were in agreement with those reported by Silver and Sonnenwirth [25]. These authors had found that gastrointestinal tract manipulation and removal prior to specimen retrieval for bacteriology markedly increased the chances of postmortem contamination of the blood. Specimen collection prior to dissection or as soon after death as possible was also strongly recommended in a study performed by Morris et al. [4].

Careful aseptic techniques are essential to minimize blood and tissue contamination during sample collection. One possible approach is obtaining blood from a peripheral vessel prior to dissection. The skin over the vessel(s) is decontaminated with disinfectants, and the blood is subsequently collected and dispensed into blood-culture bottles. An alternative, routinely used approach consists of collecting cardiac blood during autopsy post pericardium incision with heat sterilization of the heart or pulmonary artery surface and subsequent aspiration through the sterilized area. Similarly, tissue specimens for bacteriological investigations can be sampled by searing a small surface area of an organ to dryness with a red hot metal instrument. The area would then be pierced with a sterile swab or tissue blocks removed with separate sets of sterile instruments $[12,13]$. Based on the results of numerous investigations, strict aseptic approaches would allow postmortem sample contamination to be kept below $10 \%$ [4-7, 13].

The above notwithstanding, as highlighted by Tsokos and Püschel [13], specimen contamination during sampling procedures does not necessarily originate from improper or 
unscrupulous collection techniques or specimen handling. Indeed, they may also depend on the actual anatomy of the site to investigate (i.e., deeply located organs) or be due to transient/resident bacterial colonization of skin, mucosal surfaces, internal organs, or body fluids prior to death. Though improvements in sampling techniques can undoubtedly and significantly reduce the possibility of contamination during the several steps of the procedure, these precautions, however stringent, are still unable to prevent postmortem specimen contamination in its entirety.

To the best of our knowledge, the study herein presented is the first assay to compare the results of postmortem cultures performed in corpses who had undergone postmortem angiography (percutaneous access to femoral vessels after skin disinfection of the inguinal area) and corpses that had not undergone postmortem angiography (aspiration of cardiac blood from the atrium via heat-sterilized area). Despite the relatively small number of the subjects involved in this study, potentially limiting the accuracy of the research, the results of our investigations are in agreement with previous reports and allow us to conclude that:

- As far as true positives, true negatives, sample contamination, and bacterial translocation cases are concerned, accurate skin disinfection allows femoral blood to be collected and suitable for postmortem microbiological investigations with comparable results (in terms of number and type of isolated bacteria) to those obtained from cardiac blood cultures,

- Compared to the heat-sterilization technique, the percutaneous access to femoral vessels after skin disinfection is not responsible for a higher number of contaminated blood samples,

- Specimen contamination during collection can be minimized using stringent antiseptic procedures but cannot be completely avoided,

- In our case series, the majority of specimens possibly contaminated during sampling procedures cultured coagulase-negative staphylococci. These findings concur with those reported in previous studies performed on cadaveric blood and tissues,

- To conclude, postmortem angiography using a femoral approach does not constitute an impediment to the collection of peripheral blood for microbiology and vice versa. Sufficient amounts of femoral blood for bacteriology, toxicology, and biochemistry can be sampled during the same manipulation. The use of femoral blood for microbiology does not lead to an increased risk of doubtful results, which allows us to affirm that postmortem angiography can also be carried out in those cases of forensic interest that require both angiography and microbiology.

\section{References}

1. Palmiere C, Binaghi S, Doenz F, Bize P, Chevallier C, Mangin P, Grabherr S (2012) Detection of hemorrhage source: the diagnostic value of post-mortem CT-angiography. Forensic Sci Int 222:33-39

2. Grabherr S, Widmer C, Iglesias K, Sporkert F, Augsburger M, Mangin P, Palmiere C (2012) Postmortem biochemistry performed on vitreous humor after postmortem CT-angiography. Leg Med (Tokyo) 14:297-303

3. Rutty GN, Smith P, Visser T, Barber J, Amorosa J, Morgan B (2013) The effect on toxicology, biochemistry and immunology investigations by the use of targeted post-mortem computed tomography angiography. Forensic Sci Int 225:42-47

4. Morris JA, Harrison LM, Partridge SM (2006) Postmortem bacteriology: a re-evaluation. J Clin Pathol 59:1-9

5. Weber MA, Hartley JC, Brooke I, Lock PE, Klein NJ, Malone M, Sebire NJ (2010) Post-mortem interval and bacteriological culture yield in sudden unexpected death in infancy (SUDI). Forensic Sci Int 198:121-125

6. Weber MA, Klein NJ, Hartley JC, Lock PE, Malone M, Sebire NJ (2008) Infection and sudden unexpected death in infancy: a systematic retrospective case review. Lancet 371:1848-1853

7. Morris JA, Harrison LM, Partridge SM (2007) Practical and theoretical aspects of postmortem bacteriology. Curr Diagn Pathol 13:65-74

8. Tuomisto S, Karhunen PJ, Vuento R, Aittoniemi J, Pessi T (2013) Evaluation of postmortem bacterial migration using culturing and real-time quantitative PCR. J Forensic Sci 58:910-916

9. Krous HF, Beckwith JB, Byard RW, Rognum TO, Bajanowski T, Corey T, Cutz E, Hanzlick R, Keens TG, Mitchell EA (2004) Sudden infant death syndrome and unclassified sudden infant deaths: a definitional and diagnostic approach. Pediatrics 114: 234-238

10. Bajanowski T, Vege A, Byard RW, Krous HF, Arnestad M, Bachs L, Banner J, Blair PS, Borthne A, Dettmeyer R, Fleming P, Gaustad P, Gregersen M, Grøgaard J, Holter E, Isaksen CV, Jorgensen JV, de Lange C, Madea B, Moore I, Morland J, Opdal SH, Råsten-Almqvist P, Schlaud M, Sidebotham P, Skullerud K, Stoltenburg-Didinger G, Stray-Pedersen A, Sveum L, Rognum TO (2007) Sudden infant death syndrome (SIDS)-standardised investigations and classification: recommendations. Forensic Sci Int 165:129-143

11. Lobmaier IV, Vege A, Gaustad P, Rognum TO (2009) Bacteriological investigation - significance of time lapse after death. Eur J Clin Microbiol Infect Dis 28:1191-1198

12. Zanen-Lim OG, Zanen HC (1980) Postmortem bacteriology of the lung by printculture of frozen tissue. A technique for in situ culture of microorganisms in whole frozen organs. J Clin Pathol 33:474-480

13. Tsokos M, Püschel K (2001) Postmortem bacteriology in forensic pathology: diagnostic value and interpretation. Leg Med (Tokyo) 3: $15-22$

14. Grabherr S, Doenz F, Steger B, Dirnhofer R, Dominguez A, Sollberger B, Gygax E, Rizzo E, Chevallier C, Meuli R, Mangin P (2011) Multi-phase post-mortem CT angiography: development of a standardized protocol. Int J Legal Med 125:791-802

15. Palmiere C, Bardy D, Mangin P, Augsburger M (2013) Value of sTREM-1, procalcitonin and CRP as laboratory parameters for postmortem diagnosis of sepsis. J Infect 67:545-555

16. Schrag B, Roux-Lombard P, Schneiter D, Vaucher P, Mangin P, Palmiere C (2012) Evaluation of C-reactive protein, procalcitonin, tumor necrosis factor alpha, interleukin-6, and interleukin-8 as diagnostic parameters in sepsis-related fatalities. Int J Legal Med 126: $505-512$

17. Llewelyn MJ, Berger M, Gregory M, Ramaiah R, Taylor AL, Curdt I, Lajaunias F, Graf R, Blincko SJ, Drage S, Cohen J (2013) Sepsis biomarkers in unselected patients on admission to intensive or highdependency care. Crit Care 17:R60 
18. Martinez OV, Malinin TI, Valla PH, Flores A (1985) Postmortem bacteriology of cadaver tissue donors: an evaluation of blood cultures as an index of tissue sterility. Diagn Microbiol Infect Dis 3:193-200

19. Ibrahim T, Stafford H, Esler CN, Power RA (2004) Cadaveric allograft microbiology. Int Orthop 28:315-318

20. Varettas K (2013) Micro-organisms isolated from cadaveric samples of allograft musculoskeletal tissue. Cell Tissue Bank 14:621-625

21. Bettin D, Harms C, Polster J, Niemeyer T (1998) High incidence of pathogenic microorganisms in bone allografts explanted in the morgue. Acta Orthop Scand 69:311-314
22. Obeng MK, McCauley RL, Barnett JR, Heggers JP, Sheridan K, Schutzler SS (2001) Cadaveric allograft discards as a result of positive skin cultures. Burns 27:267-271

23. Babb JR, Hall AJ, Marlin R, Ayliffe GA (1989) Bacteriological sampling of postmortem rooms. J Clin Pathol 42:682-688

24. Søgaard P, Larsen KE, Buhl L, Lou HE, Henriques U (1991) Bacteriological autopsy. I. A methodological study. APMIS 99:541-544

25. Silver H, Sonnenwirth AC (1969) A practical and efficacious method for obtaining significant postmortem blood cultures. Am J Clin Pathol 52:433-437 